\title{
La igualdad de género en la docencia universitaria: transitando de la universalidad a la especificidad
}

\author{
Gender equality in university teaching: from universability to specificity
}

ISSN 2071-8748

E-ISSN 2218-3345

(c) (1) $\$$

BY NC SA

URI: http://hdl.handle.net/11298/901

DOI: http://dx.doi.org/10.5377/entorno.v0i66.6738

\author{
Gladys Merma-Molina \\ ORCID: 0000-0002-9856-6314 \\ María Alejandra Ávalos-Ramos
ORCID: 0000-0002-4925-9174 \\ María Ángeles Martínez-Ruiz \\ ORCID: 0000-0001-6028-1877 \\ Universidad de Alicante \\ gladys.merma@ua.es \\ Recibido: 29/07/18 \\ Aprobado: 12/12/18
}

\section{Resumen}

El objetivo de este estudio fue analizar si se incluye o no, y cómo se integra en su caso, el significado y alcance de la igualdad de género entre mujeres y hombres en la docencia universitaria. En la investigación, con enfoque cualitativo, participaron 25 profesores de los siete centros de la Universidad de Alicante. El instrumento utilizado para la recolección de datos fue una entrevista narrativa diseñada ad hoc para el estudio. El análisis de los datos se realizó con el software Aquad 7. Los hallazgos destacan que el profesorado considera que el enfoque de género es relevante en la docencia universitaria, aunque hay una considerable presencia de voces que señalan que no lo es, e incluso afirman que le quita calidad a los contenidos de las materias que imparten. Asimismo, los docentes que lo integran o están a favor de ello o arguyen que no requieren formación específica, aunque contradictoriamente destacan la existencia de factores limitantes como la complejidad y el desconocimiento del tema y la falta de referentes femeninas en su ámbito

\section{Abstract}

The purpose of this study was to analyze whether to include or not, and how would integrate, in this case, the meaning and outreach of gender equality among women and men in the teaching endeavor. Twenty five professors from the seven centers of Universidad de Alicante (Alicante's University) participated in this qualitative focused investigation. An ad hoc designed descriptive interview was the instrument used for this study. The Aquad 7 software was used for data analysis. The findings reveal that professors consider that gender approach is important in the university teaching endeavor, although there is a considerable presence of voices pointing that it is not, and even affirm that it diminishes the quality of the courses that they teach. In like manner, the staff conforming professors or those in favor argue that they do not require specific instruction, however, they contradictorily highlight some intervening restrictive factors such as the complexity and unawareness on the subject and the lack of feminine 
de conocimiento. Se concluye que la incorporación de la perspectiva de género no ha llegado a las aulas universitarias, que el marco legislativo en materia de género no ha tenido resultados efectivos ni funcionales, que hay una resistencia del profesorado para su inclusión en la docencia, y que la integración del género obedece a la voluntad solo de algunos profesores que están parcialmente convencidos de su beneficio y necesidad.

\section{Palabras clave}

Educación superior; Desarrollo profesional; Planes de estudio; Personal docente; Igualdad social. referents in their knowledge scope. It may be said that the inclusion of gender approach has not reached the university classrooms; that the legal framework dealing with the gender issue has not produced neither effective nor functional results; that staff professors resent its inclusion in the teaching process and that gender inclusion rests on the will of only some professors who are partially convinced of its advantages and need.

\section{Keywords}

Higher education, Professional development, Study programs, Social equality.

\section{Introducción}

Las investigación sobre las inequidades de género en la Educación Superior en España ha mostrado un cierto avance; sin embargo, estos progresos han sido superficiales y aún no han llegado a las aulas universitarias, donde los conocimientos que se transmiten carecen de este enfoque (Merma, Ávalos, \& Martínez, 2017). La legislación del Estado español aboga por incluir en todos los niveles educativos la perspectiva de género, la igualdad y la no discriminación; no obstante, la Ley Orgánica 3/2007, de 22 de marzo, para la igualdad efectiva entre hombres y mujeres no parece haber tenido el impacto deseado, por lo que podría decirse que su aplicación ha sido más formal que sustancial.

Ballarín (2013) indaga si hay resistencias del profesorado para incluir el feminismo académico en los programas docentes y concluye que hay creencias y prejuicios como el hecho que no se reconozca la existencia de un saber específico de y sobre las mujeres que explique cómo se reproducen y difunden las desigualdades, que hay una defensa de que las relaciones discriminatorias se producen de forma natural, que el profesorado considera que no necesita formación especializada y que piensan que no son necesarias asignaturas específicas. Por su parte, GarcíaPérez et al. (2011) demuestran que los estudiantes tienen un insuficiente grado de conocimientos básicos sobre el género y es que "estos contenidos solo son abordados en el plan de estudios a voluntad libre del profesorado más sensibilizado" (p. 21). Los autores arguyen que la realidad educativa universitaria muestra que no es habitual encontrar asignaturas que aborden el género como un eje central y que esto solo ocurre en materias específicas optativas.

\section{La institucionalización del enfoque de género en la Educación Superior}

La incorporación de la perspectiva de género en la educación se ha articulado con los objetivos de la igualdad de género planteadas en el Marco de Acción de Dakar que manifiesta el compromiso de todos los países por lograr una Educación para todos y con los Objetivos de Desarrollo del Milenio adoptados por la ONU, medidas que si bien son universales, no han sido asumidas a través de la doctrina epistemológica del empirismo.

Santos, Nunes y Meneses (2007) sostienen que a esta postura universal le hace falta la idea de una ecología de conocimientos y diálogo epistémico que permitan conceptualizaciones y prácticas locales $-\mathrm{y}$ menos globales- para trabajar la igualdad de género. En esta misma línea, algunos académicos (por ejemplo, Tembon \& Fort, 2008) han argumentado que este enfoque universalista ha contribuido solo a que más niñas vayan a la escuela y otros autores (por ejemplo, Aikman \& Unterhalter, 2005; Baltliwala, 1994; Chisamya, DeJaeghere, Kendall, \& Khan, 
2012, DeJaeghere, 2015; Unterhalter, 2014) han cuestionado la estrecha conceptualización del predominio de las mujeres en el enfoque de desarrollo que no necesariamente aborda la discriminación de género reproducida en las prácticas diarias, en las escuelas y la falta de un enfoque en la transformación social de las familias y de las sociedades.

Como argumenta Kabeer (2005), las desigualdades de género son multidimensionales y no pueden reducirse a un conjunto de prioridades universales. En esta misma línea, Santos (2012) sugiere que debemos buscar los significados y las acciones en las prácticas locales que tienen la posibilidad de transformación; es decir, mecanismos institucionales e individuales fomentados a través de procesos pedagógicos y prácticas locales.

Donoso-Vázquez y Velasco-Martínez (2013) sostienen que en la educación, "al ser una disciplina que se aplica e influye en la realidad, el enfoque de género cobra una importancia más acusada como medio para analizar y transformar la realidad" (p. 76); sin embargo, la enseñanza del género no es una práctica sencilla porque el propio docente es una persona con género, que está situado en un entorno académico o de aprendizaje que también está relacionado con el género (Carøe, Thidemann, \& Højlund, 2016). Esto implica que a menos que el docente reflexione críticamente sobre quién es y lo que trae a la situación enseñanza (McNeil, 2012) "puede tropezar con sus propios puntos ciegos" (Carøe, Thidemann, \& Højlund, 2016, p. 4); en consecuencia los ámbitos privado y profesional del profesor, al tener género, podrían influir en su forma de enseñar.

Buquet (2011) sostiene que la incorporación del género en los currículos es un factor clave para el proceso de institucionalización de la perspectiva de género en las universidades que tiene dos objetivos: impactar en la preparación académica de las y los jóvenes proporcionándoles nuevos elementos teóricometodológicos para la comprensión de la realidad social, y promover su formación para la deconstrucción de diversas formas de discriminación imperantes en la sociedad y transmitir valores de igualdad y de respeto a las diferencias.

Algunos autores (por ejemplo, Cassese, Holman, \& Schneider, 2015; Donoso-Vázquez, Montané, \& Pessoa, 2014) han abogado por la transversalización del género en la Educación Superior como una forma de cambiar los roles tradicionales y eliminar las desigualdades. Defienden la idea de incorporar el género en los contenidos temáticos de las asignaturas, en los recursos didácticos, en el uso del lenguaje inclusivo y no sexista, en la política y gestión institucional y en la cotidianidad de la vida universitaria transformando los sistemas discriminatorios en espacios de igualdad; es decir, como una estrategia metodológica y política (Chaves, 2015) que parte de la concienciación del profesorado (García-Pérez et al., 2011).

Con base en este marco, el objetivo de este estudio es analizar si se incluye o no - y cómo se integra en su caso-el significado y alcance de la igualdad de género entre mujeres y hombres en la docencia universitaria, en la Universidad de Alicante (España). Las cuestiones de investigación que han orientado el estudio son: ¿cómo y cuándo, el profesorado universitario, integra el enfoque de género en su docencia?, ¿cómo recibe el alumnado la incorporación del enfoque de género en la docencia?, ¿qué dificultades y problemáticas tiene el docente para integrar la igualdad de oportunidades de género en la docencia?, ¿cuáles son las percepciones del profesorado universitario sobre la importancia de incluir el enfoque de género en su docencia?, ¿cómo se podría mejorar la integración de la igualdad de oportunidades de género en la docencia universitaria?

\section{Metodología}

\section{Participantes}

En el estudio se adoptó el enfoque cualitativo (Denzin \& Lincoln, 2012). La investigación se ha realizado con base en muestreo intencional (Glaser \& Strauss, 1967; Patton, 1990), invitándose a participar en el estudio al profesorado de las siete Facultades de la Universidad de Alicante. En la investigación se presenta el análisis de 25 entrevistas en profundidad (10 hombres y 15 mujeres) de una muestra de profesorado de las siguientes Facultades: Filosofía y Letras (5), Educación (7), Derecho (3), Ciencias de la Salud (2), Económicas y Empresariales (3), Escuela Politécnica Superior (2), Facultad de Ciencias (3).

La edad media de los participantes es de 42-43 años, siendo el más joven de 33 años y el más adulto de 69 años. Respecto a los años de experiencia, hay un profesor que tiene entre 0-5 años, 12 entre 6-15 años, 5 entre 16-25 años y 7 profesores que tienen una experiencia laboral de más de 25 años. 


\section{Instrumento}

La herramienta empleada para la recolección de los datos es la entrevista narrativa, que es especialmente útil en la investigación educativa (Coulter \& Smith, 2009). El instrumento fue diseñado ad hoc para este estudio teniendo en cuenta la literatura experta de Ballarín (2013), Buquet (2011), Cassese, Bos y Duncan (2012), Donoso-Vázquez y Velasco-Martínez (2013), Foster, Kerr, Hopkins, Byrne y Ahall (2013), García-Pérez et al. (2011) y Grünberg (2011), y de otros estudios realizados en la Red de Investigación Diseño y Atención a las Oportunidades de Género en la Educación Superior (Martínez, Merma \& Gavilán, 2013; MermaMolina, Ávalos, \& Martínez, 2015; Merma-Molina, Ávalos, \& Martínez, 2017).

El objetivo de la entrevista es analizar si se incluye o no de forma específica o transversal el significado y alcance de la igualdad entre mujeres y hombres en la docencia universitaria en las siete Facultades de la Universidad de Alicante. La validación del contenido de la entrevista fue realizada por cinco profesores, expertos, que pertenecen a la Red de Investigación Diseño y Atención a las Oportunidades de Género en la Educación Superior (https://web.ua.es/es/ proyectogenero/somos-el-proyecto-genero.html).

La entrevista está constituida por un apartado donde se recogen los datos sociodemográficos de los participantes y cinco cuestiones en las que se indagan:

1. Cómo y cuándo se integra la igualdad de oportunidades de género en la docencia de las asignatura: en el temario, como contenido específico, como actividad específica, como temática transversal, etc.

2. Cómo recibe el alumnado la incorporación de la perspectiva de género en la docencia, cuáles son sus actitudes y opiniones.

3. Qué dificultades o problemáticas encuentra el profesorado universitario para integrar la igualdad de oportunidades de género en la docencia.

4 Por qué cree el profesorado universitario que es importante incluir el enfoque de género en la docencia universitaria.

5. Cómo se podría mejorar la inclusión de la igualdad de oportunidades de género en la docencia universitaria, desde la perspectiva del profesorado.

\section{Procedimiento}

Para la recolección de datos, se hizo extensiva la invitación al profesorado de los diferentes centros de la Universidad de Alicante a quienes se les explicó los objetivos de la investigación; accedieron a participar en el estudio 25 profesores.

Las entrevistas se concertaron y realizaron durante el segundo cuatrimestre del curso académico 2017-2018, en los despachos del profesorado en la propia universidad, según su disponibilidad de tiempo. Luego, el equipo investigador efecctuó la transcripción de las narrativas en formato RTF. Posteriormente, la información recogida se analizó con el software informático cualitativo AQUAD 7 (Huber, 2012). Este programa nos ha permitido almacenar las unidades de significado en una base de datos después de un proceso de recopilación, análisis y edición. De manera concreta, el procedimiento consistió en realizar, en primer lugar, una revisión inductiva (Maykut \& Morehouse, 1994) del contenido de las narrativas para establecer las primeras conexiones entre las cuestiones de investigación y las temáticas emergentes de este estudio.

Se crearon los códigos inferenciales, categorizándose y validándose siguiendo un proceso de triangulación en el que intervinieron tres profesoras universitarias especialistas en géneroy educación de la Red de investigación en diferenciales de género de la Universidad de Alicante (España). Por último, se diseñó un mapa de códigos definitivo del que emergieron las cinco temáticas fundamentales de este estudio:

\section{Resultados y discusión}

El análisis de los resultados se presenta en función de las frecuencias absolutas (FA), siendo estas el número de veces de aparición de un concepto en las narrativas y, por otro lado, el porcentaje de las frecuencias absolutas (\%FA), que representa su total (FA.100/total FA).

Los resultados que se exponen a continuación se refieren a las aportaciones extraídas de los profesores universitarios sobre la temática de la inclusión de la perspectiva de género en la docencia universitaria.

Respecto a la cuestión sobre la integración de la igualdad de oportunidades de género en la docencia (Tabla 1), los hallazgos indican que el profesorado afirma que sí 
la incluye (60\%) en sus asignaturas. Esta integración la realizan atendiendo a la sensibilidad del lenguaje (23.33\%), planteándola de forma transversal (21.66 \%) e incluyendo el enfoque de género en algún tema específico dentro de sus asignaturas (15\%). Por otro lado, emergen manifestaciones que reflejan que el profesorado no aborda ( $40 \%)$ ni incluye esta perspectiva de ninguna forma. Todos estos hallazgos quedan representados en los siguientes fragmentos manifestados por algunos docentes:

Tenemos mucha sensibilidad en diferenciar en el alumnado o en el estudiantado, aquellos conceptos que podrían dar algún tipo de interpretación o mala interpretación sexista al respecto (Pro-06).

Nosotros lo hacemos de forma transversal. Como temática transversal siempre surge el tema, casi día a día (Pro-25).

Hay una parte específica del temario que trata sobre el entrenamiento de la mujer, es decir la planificación del entrenamiento en función del ciclo menstrual (Pro-11). Realmente nosotros no tenemos una política específica de cómo abordar la problemática de las igualdades de género. No tenemos incluido ningún tipo de referencia al respecto (Pro-03).

Tabla 1. Integración del enfoque de género en la docencia

\begin{tabular}{|l|r|r|r|}
\hline \multicolumn{2}{|l|}{ Códigos } & FA & $\%$ FA \\
\hline Sí & Sensibilidad del lenguaje & $\mathbf{7 2}$ & $\mathbf{6 0 \%}$ \\
\hline \multirow{3}{*}{} & Transversal & 28 & $23.33 \%$ \\
\cline { 2 - 4 } & Tema especifico & 26 & $21.66 \%$ \\
\hline \multirow{2}{*}{ NO } & 18 & $15 \%$ \\
\hline & & $\mathbf{4 8}$ & $\mathbf{4 0} \%$ \\
\hline
\end{tabular}

Las reacciones que el profesorado observa en los estudiantes cuando aborda la perspectiva de género en sus clases (Tabla 2) se refieren, mayoritariamente, a percepciones de los alumnos concienciados con el tema $(61.72 \%)$; por otro lado, perciben un alumnado con actitud de indiferencia en cuestiones de género (19.75\%), y en menor grado, se encuentran con estudiantes que se sorprenden al abordar esta temática (9.87\%); finalmente, en otros casos, el alumnado difiere en sus opiniones sobre esta perspectiva (8.64 \%). Las siguientes narrativas evidencian estas situaciones:

Yo creo que la tónica general es normalidad, concienciación, o sea no hay nadie que le extrañe y a las chicas al contrario les produce un efecto positivo, un refuerzo en el aprendizaje (Pro-12).
No veo al alumnado demasiado concienciado; no están alerta sobre el tema. Si les haces la reflexión, la entienden, pero no sale de ellos ver la diferencia de oportunidades que existe entre varones y mujeres (Pro09).

En el caso de la actividad física y del deporte sí que les resulta curioso que se vea más clara la incorporación de las chicas a deportes como el fútbol; pero se sorprenden cuando tratamos la incorporación del hombre a disciplinas como la gimnasia rítmica (Pro-13). Se genera mucho debate al trabajar estos temas; sí que es verdad que cada uno hace una interpretación y se crean diferentes opiniones, sobre todo últimamente con el uso del lenguaje por ejemplo el uso de "estimados" $y$ "estimadas", hay alumnos que les resulta redundante y a otros les parece bien (Pro-04). 
Tabla 2. Percepción del profesorado sobre las reacciones del alumnado universitario

\begin{tabular}{|c|c|c|}
\hline Códigos & FA & $\% \mathrm{FA}$ \\
\hline Concienciación con la temática & 50 & $61.72 \%$ \\
\hline Indiferencia & 16 & $19.75 \%$ \\
\hline Sorpresa & 8 & $9.87 \%$ \\
\hline Diferencias de opiniones & 7 & $8.64 \%$ \\
\hline Total & 81 & \\
\hline
\end{tabular}

En lo concerniente al segundo actor educativo —el alumnado universitario-, en general, este está concienciado con la temática (61.72\%), aunque también se percibe una presencia destacable $(19.75 \%)$ de indiferencia en ellos, por lo que la sensibilización en el enfoque de género en la educación universitaria sigue siendo una tarea pendiente. La formación de género en la universidad es importante porque es en esta institución donde se dota al alumnado de un marco interpretativo de la realidad que encaja con elementos de justicia social y con la construcción de un mundo más equitativo y humano (Donoso-Vázquez \& Velasco-Martínez, 2013).

En lo que respecta a las dificultades que los profesores universitarios encuentran para introducir la igualdad de oportunidades en sus aulas (Tabla 3), algunas de las reflexiones indican que los docentes no tienen dificultades $(48,25 \%)$. En contraposición, aparecen afirmaciones que señalan problemáticas asociadas a la excesiva complejidad que este tema les supone $(20 \%)$, al desconocimiento sobre la materia (18.57\%) y finalmente, indican como problemática la escasez de referentes femeninos (12.85 \%) en todos los ámbitos. Estos obstáculos se reflejan en los siguientes fragmentos:
No encuentro ninguna dificultad, al contrario es un contenido que hay que desarrollar. Hace 20 años era un contenido poco investigado, pero con el paso de los años hay muchísima información teórica y práctica. Hay mucha literatura el que no lo trabaja es porque no quiere (Pro-15).

Luego es complicado, como entrenadoras cuesta muchísimo introducir la idea de que las mujeres podemos ser entrenadoras no solamente de mujeres, sino también de hombres. Hay muy pocos ejemplos de entrenadoras y eso dificulta mucho (Pro-11).

Hay profesionales que no se reciclan y entonces sí que les falta ese conocimiento (Pro-23).

También es verdad que como docentes en la universidad en nuestra área somos muchos más hombres que mujeres y ahí sí que hace falta que esto cambie, que haya más presencia de mujeres (Pro-01).

Claro, también hay muy pocos créditos en el Máster de Cooperación Internacional, no les da tiempo ha interiorizar lo que significa una función profesional. Tendrían que ver no solo cómo se proponen esas políticas, sino vigilar si se cumplen (Pro-02). 
Tabla 3. Problemáticas y dificultades

\begin{tabular}{|l|l|r|r|}
\hline \multicolumn{2}{|l|}{ Códigos } & FA & $\%$ FA \\
\hline \multirow{2}{*}{ Sí } & & $\mathbf{4 0}$ & $\mathbf{5 7 . 1 4} \%$ \\
\hline \multirow{5}{*}{ NO } & Complejidad del tema & 14 & $20 \%$ \\
\cline { 2 - 4 } & Desconocimiento del tema & 13 & $18.57 \%$ \\
\cline { 2 - 4 } & Falta de referentes femeninas & 9 & $12.85 \%$ \\
\cline { 2 - 4 } & Falta de créditos específicos & 4 & $5.71 \%$ \\
\hline & & 30 & $\mathbf{4 2 . 8 5 \%}$ \\
\hline
\end{tabular}

A pesar de que se ha profundizado en la investigación en el ámbito de profesión docente (Concepción, 2010; Domínguez, 2015; Miranda, 2016; Pulido, 2014), donde se ha hecho énfasis en el desarrollo profesional, el profesorado universitario, que tiene la misión de formar profesionales que en el futuro serán responsables de diseñar, poner en práctica y evaluar programas y políticas públicas humanas y justas en los distintos sectores de la vida económica política y social de país, tiene limitaciones para integrar el enfoque de género en su docencia, lo que restringe su desempeño profesional y pedagógico para el logro de prácticas inclusivas, de igualdad y de derechos de las personas (Cotelo-Armenteros, Riol-Hernández, \& RomeroViamonte, 2018). Estas limitaciones se reflejan en el hecho de que el profesorado que integra el enfoque de género en su docencia o que está a favor de hacerlo manifiesta que tiene dificultades, siendo los problemas más acuciantes la complejidad del tema, la falta o escaso dominio del enfoque de género y el desconocimiento de referentes femeninas en su ámbito de conocimiento.

Con relación a la importancia de la inclusión del enfoque de género en la docencia (Tabla 4), las narrativas destacan la relevancia de la temática para el profesorado (85.47\%) ya que le resulta fundamental para fomentar la igualdad de oportunidades (29.03\%), para generar conciencia social $(24.19 \%)$, desarrollar la perspectiva conceptual y la transferencia de conceptos (12.90\%), visibilizar a la mujer (11.29 \%) y darle importancia enfocando el tema de forma transversal $(8,06 \%)$.
Si bien el enfoque de género en la docencia universitaria es relevante según las percepciones del profesorado, existe una presencia nada desdeñable de manifestaciones que señalan que no lo es (14.51\%); consideran que la temática como secundaria e incluso manifiestan que su integración en la docencia le quitaría valor al conocimiento científico de $\mathrm{la} / \mathrm{s}$ materia/s que imparten. Las siguientes manifestaciones reflejan estos hallazgos:

Es muy importante incluir esta perspectiva porque son los y las futuros y futuras docentes y se debe trabajar y fomentar la igualdad de oportunidades desde las aulas (Pro-07).

Es fundamental incluir el enfoque para alcanzar una sociedad de plena igualdad como es lo deseable, en todos sus aspectos. Fomentar esa conciencia (Pro-08). Lo consideramos importantísimo, este año hemos hecho un pequeño esfuerzo y hemos sacado figuras femeninas, autoras femeninas de la traducción que hasta este momento no lo habíamos hecho (Pro-19). Es fundamental incluirlo por la transferencia que tendrá después en todas las facetas del alumnado, en su profesión (Pro-02).

No lo veo importante. No es para darle un formato oficial, como diríamos que todas las asignaturas tengan que tener una orientación a la igualdad de género; no es necesario. Pienso que esto no solo me quitaría tiempo para abordar los contenidos, sino que la asignatura podría ser vista escasa e insuficiente (Pro-20). 
Tabla 4. Relevancia del enfoque de género en la docencia universitaria

\begin{tabular}{|l|r|r|r|}
\hline \multicolumn{2}{|l|}{ Códigos } & FA & $\% \mathrm{FA}$ \\
\hline Sí & Igualdad de oportunidades & 18 & $\mathbf{8 5 . 4 7 \%}$ \\
\hline \multirow{5}{*}{} & Conciencia social & 15 & $29.03 \%$ \\
\cline { 2 - 4 } & $\begin{array}{l}\text { Perspectiva conceptual y } \\
\text { transferencia }\end{array}$ & 8 & $12.90 \%$ \\
\cline { 2 - 4 } & Visibilidad de la mujer & 7 & $11.29 \%$ \\
\cline { 2 - 4 } & De forma transversal & 5 & $8.06 \%$ \\
\hline No es importante & 9 & $14.51 \%$ \\
\hline & & 62 & \\
\hline
\end{tabular}

Como complemento a lo anterior y aunque los docentes son conocedores de que para avanzar en la igualdad hay que generar la conciencia social y visibilizar a la mujer, no consideran necesario incorporar el enfoque de género en todas las asignaturas, sino solo en algunas como en Ciencias Sociales y en Humanidades y, además, hay una escasa presencia de opiniones a favor de la transversalización del género en la docencia.

Estas posturas no son concordantes con las conclusiones de Cotelo-Armenteros, Riol-Hernández y Romero-Viamonte (2018), y Naranjo (2010) que arguyen a favor de transversalizar el enfoque de género en la docencia universitaria puesto que posibilitaría la ventaja de elevar la profesionalización de los egresados de cualquier especialidad para convertirlos en agentes de cambio activo, capaces de prevenir o enfrentar con éxito problemas como la discriminación o la violencia de género; en suma, hace falta una profesionalización en género de la Educación Superior que se efectúe tanto en los grados como en los postgrados.

Por último, las propuestas de mejora (Tabla 5) que expusieron los participantes del estudio se referían a la importancia de la formación en género especialmente en la Educación primaria (22.10\%), esto es educar en etapas previas a la universitaria y educar en las familias. También aparecen propuestas relacionadas con la formación específica (20 \%) a través de cursos, talleres o seminarios dirigidos tanto al profesorado como a los estudiantes. Con igual presencia, emergen las propuestas referidas a incluir asignaturas específicas en los planes de estudio así como revisarlos y modificarlos (13.68 \%).

Asimismo, los docentes proponen abordar la perspectiva de género de manera transversal (12.63\%), dando mayor visibilidad a la mujer desde los diferentes ámbitos dentro de la universidad (10.52\%), fomentando la investigación de género $(4.21 \%) y$, finalmente, se presenta una postura totalmente contraria que plantea que no se deben abordar ni incluir temáticas concretas de género (3.15\%). Así manifiestan los docentes:

Yo creo que es una responsabilidad de la educación de niños y niñas, veo efectivamente que hay que empezar desde abajo y luego, pues continuar (Pro-08).

Yo pondría alguna asignatura de Políticas transversales de género (Pro_01).

Bueno yo creo, y mi visión del tema es que se revisen y modifiquen los planes de estudio que se hagan en un futuro y que se introduzca la mención de las Políticas de Género (Pro-16).

Proponer cursos y temas transversales; bajo mi experiencia, no tendría que ser un tema específico de una asignatura. Hay que fomentar esa sensibilidad en el alumnado (Pro-22). 
Después de esta entrevista, donde me has hecho reflexionar más sobre el tema, creo que habría que incorporar talleres en nuestras asignaturas, donde se trabaje explícitamente la igualdad de género, que no quede solo en el currículo oculto (Pro-13).

Dar protagonismo y visibilidad a la mujer, por ejemplo, en mi asignatura que está más ligada a hombres, pues es interesante que haya una mujer dándola (Pro-17).
La investigación al final es lo que provoca el prestigio, cuando se realizan investigaciones, datos científicos sobre el tema y este es un ítem muy importante para avanzar (Pro-04).

Mi propuesta es que en las asignaturas se traten temas académicos únicamente, sin tener en cuenta otros factores como la igualdad de género (Pro-05).

Tabla 5. Propuestas de mejora

\begin{tabular}{|c|c|c|}
\hline Códigos & FA & $\% \mathrm{FA}$ \\
\hline Educación Primaria & 21 & $22.10 \%$ \\
\hline Formación específica & 19 & $20 \%$ \\
\hline Incluir asignaturas especificas & 13 & $13.68 \%$ \\
\hline Revisión y modificación de los planes de estudio & 13 & $13.68 \%$ \\
\hline Tema transversal & 12 & $12.63 \%$ \\
\hline Dar mayor visibilidad a la mujer desde la universidad & 10 & $10.52 \%$ \\
\hline Investigación específica & 4 & $4.21 \%$ \\
\hline No abordar el género & 3 & $3.15 \%$ \\
\hline Total & 95 & \\
\hline
\end{tabular}

Frente a las problemáticas señaladas, el profesorado alude a la formación específica como una propuesta de mejora, aunque, prioritariamente, recomienda la formación en género en otras etapas educativas, ya que, según ellos, es donde el alumnado debe recibir esta formación y no en la universidad. El profesorado universitario percibe que no necesita ningún tipo de formación, aunque luego, contradictoriamente, no se ve capacitado para incorporar el enfoque de género en su docencia.

¿Qué hacer para mejorar la integración del género en la docencia universitaria?

La universidad tiene la responsabilidad de contrarrestar los efectos negativos de la cultura patriarcal y de evitar cualquier forma de exclusión que se genere a partir de los estereotipos de género (Cháves, 2015) y, en este proceso, la función del profesor es crucial. El sistema educativo universitario, como el contexto de socialización secundaria más importante, tiene el rol protagónico en la transmisión de modelos sociales, en la reproducción de valores, normas, creencias, actitudes que se expresan de modo implícito y explícito en las nociones culturales que se transmite por medio del currículo.

La ausencia en el currículo de conocimientos aportados por la investigación en la perspectiva de género, de interés científico, social y educativo, no solo está dificultando en el alumnado universitario la comprensión de una realidad donde hay inequidades, sino que contribuye a la naturalización de los prejuicios sexistas. Para atajar esta problemática, el profesorado ha de integrar la perspectiva de género en su docencia en diferentes niveles en el currículum, como la transversalidad, las asignaturas específicas, los módulos y las actividades específicas. La introducción del enfoque de género en la docencia pasa por cuatro niveles: 
- Primer nivel: desarrollar la conciencia de género, que tiene que ver con la adquisición de elementos conceptuales que den nombre a las distintas realidades vinculadas con la igualdad y con las inequidades. El "dar nombre" conlleva a saber identificar y analizar críticamente la realidad (Donoso-Vázquez \& Velasco-Martínez, 2013); es importante poner nombre a situaciones de discriminación por género.

- Segundo nivel: pasar a una conciencia crítica, un cuestionamiento personal sobre la internalización y reproducción de estereotipos de género (Giraldo \& Colyar, 2012) y de los mitos que los refuerzan. El análisis introspectivo permite adquirir una conciencia crítica hacia las propias actuaciones y ser capaces de analizar otras situaciones ajenas.

- Tercer nivel: se refiere a la adquisición de herramientas y a la capacitación para hacer un análisis de la realidad desde una mirada que se acerca y pone en práctica la perspectiva de género; es decir se refiere a la predisposición para la acción a partir del análisis crítico (Colás \& Jiménez, 2006; Donoso-Vázquez \& Veslasco-Martínez, 2013). La conciencia crítica es necesaria para fomentar la propia conciencia de género y al mismo tiempo comprometerse como agentes activos de la transformación de normas sociales (Rebollo, García, Piedra, \& Vega, 2011).

- Cuarto nivel: se refiere a la transformación del entorno educativo y de las disciplinas y materias a través de nuevos modelos de docencia más inclusivos. En este nivel hay que hacer referencia a la elaboración de propuestas y estrategias de cambio en la docencia, como por ejemplo:

- Desarrollar investigaciones y prácticas educativas orientadas a reconstruir una educación que promueva una visión más igualitaria, en lugar de reproducir acríticamente las desigualdades.

- Contrarrestar la naturalización de las percepciones sobre lo que puede ser un hombre o una mujer que posiblemente subyacen en los contenidos de las materias que se imparten. La condición de género no está dada por la naturaleza biológica del sexo de la persona; esta posee un carácter socialmente construido que hay que explicar y develar en sus contradicciones.
- Considerar los diferentes intereses, valores y necesidades emocionales de cada alumno y alumna.

- Promover la corresponsabilidad familiar en la docencia, ya que las mujeres generalmente llevan sobre sus espaldas el peso del trabajo doméstico.

- Elaborar metodologías adecuadas para que los estudiantes adquieran experiencias y capacidades que tradicionalmente han sido adjudicados a uno de los sexos de manera casi exclusiva.

- Evitar temas y ejemplos estereotipados por género y seleccionarlos en función de si responden a los intereses de todos y de todas.

- Incluir las experiencias y aportes de las mujeres en la ciencia.

\section{Conclusiones}

En la teoría, se sostiene que el género y la diversidad en la Educación Superior son temáticas muy recurrentes, confluyentes y que están integradas (Caroe, Thidemann, \& Hojlund, 2016), pero los hallazgos del estudio muestran que en la realidad esto no es del todo cierto. A partir del análisis de los hallazgos se concluye que la incorporación del género en la docencia universitaria es parcial y no se realiza de forma planificada, sistemática, específica, ni transversal, siendo una de las causas destacables la resistencia del profesorado.

Si bien existe cierta sensibilidad y comprensión de la igualdad de género, sigue siendo un desafío para el profesorado no saber cómo aplicar este enfoque en su docencia, lo que limita su desempeño profesional pedagógico. Se evidencian carencias en el proceso de sistematización metodológica de los contenidos en temas de género, insuficiente aprovechamiento de las potencialidades educativas de las materias para el tratamiento de la equidad de género, así como una escasa preparación y proyección del profesorado para la educación en género. No existen acciones concretas ni estrategias para integrar el género en los contenidos, que impulse la creación de un currículo sensible a las desigualdades y que promueva la igualdad. 
Asimismo, el marco legislativo no ha tenido resultados efectivos ni funcionales y actualmente la docencia específica y la formación en género obedecen a la voluntad de algunos profesores que están parcialmente convencidos de su beneficio y necesidad. Los planes docentes no incluyen la perspectiva de género $y$, consecuentemente, el género sigue siendo tratado simplemente en espacios con reflexiones poco profundas, con debates sobre ideas y enfoques que muchas veces se dejan en silencio o se hacen aisladamente, sin planificación, sin diálogo, sin respuestas coordinadas, como simples prácticas no documentadas a menudo invisibles dentro de la vida académica cotidiana (Ballarín, 2013; Carøe, Thidemann, \& Højlund, 2016).

La sensibilización del género, superficial y generalista, tanto en el profesorado como en el alumnado, no es suficiente para lograr la igualdad (Cháves, 2015), esta solo es una parte del proceso; lo que realmente proporciona buenos resultados es el manejo instrumental teórico-práctico del análisis del género. Por tanto, incorporar el género en la docencia universitaria no solo implica el reconocimiento de los diferentes roles del género en los contextos en que nos desarrollamos y la forma en que estos determinan mecanismos de inclusión o exclusión en cuanto al acceso, uso y control de los recursos existentes y de los beneficios que se produzcan.

La docencia con enfoque de género ha de ir más allá de la sensibilización y de desarrollar actividades para mujeres o usar un cierto tipo de lenguaje en la docencia; implica un enfoque teórico transformador, la elaboración de estrategias y el diseño de propuestas de acciones tanto para mujeres, hombres y personas de otros géneros, considerando sus necesidades e intereses desde la diversidad de sus identidades de género y de sus condiciones concretas.

\section{Referencias}

Aikman, S., \& Unterhalter, E. (Eds.) (2005). Beyond Access: Transforming Policy and Practice for Gender Equality in Education. Oxford: Oxfam.

Ballarín, P. (2013). Docencia universitaria y conocimientos en torno al género. Resistencias, creencias y prejuicios. Cuestiones de Género: de la Igualdad y la Diferencia, 8, 89-106.
Batliwala, S. (1994). The meaning of women's empowerment: New concepts from Action. En G. Sen, A. Germain, \& L. Chen (Eds.), Population policies reconsidered. Health, empowerment, and rights (pp. 127-138). Boston: Harvard School of Public Health.

Buquet, A. G. (2011). Transversalización de la perspectiva de género en la educación superior: Problemas conceptuales y prácticos. Perfiles educativos, 33(SPE.), 211-225.

Carøe, C., Thidemann, S., \& Højlund, D. (2016). Introduction: Teaching Gender and Diversity in Higher Education. Kvinder, Køn \& Forskning, 25(1), 3-6.

Cassese, E. C., Bos A. L., \& Duncan, L. E. (2012). Integrating gender into the political science core curriculum. PS: Political Science and Politics, 45(2), 238-243.

Cassese, E., Holman, M., Schneider, M. C., \& Bos, A. L. (2015). Building a Gender and Methodology Curriculum: Integrated Skills, Exercises, and Practices. Journal of Political Science Education, 11(1), 61-77.

Cháves, R. (2015). Aspectos relevantes para la transversalización de la perspectiva de género en el proceso de desarrollo curricular universitario. Revista ESPIGA, 29, 33-43.

Chisamya, G., DeJaeghere, J., Kendall, N., \& Khan, M. A. (2012). Gender and education for all: Progress and problems in achieving gender equity. International Journal of Educational Development, 32(6), 743-755.

Colás, M. P., \& Jiménez, R. (2006). Tipos de conciencia de género del profesorado en los contextos escolares. Revista de Educación, 340, 415-444.

Concepción, P. (2010). El desarrollo profesional docente del profesorado novel de la Universidad de Ciego de Ávila (Tesis doctoral). Universidad de Granada, Granada.

Cotelo-Armenteros, S., Riol-Hernández, M., \& RomeroViamonte, J. (2018). La profesionalización docente en género: contribución al referente de género del profesorado universitario. Educación y Sociedad, 16(1), 95-107.

Coulter, C. A., \& Smith, M. L. (2009). The construction zone: Literary elements in narrative research. Educational Researcher, 38(8), 577-590.

DeJaeghere, J. (2015). Reframing gender and education for the post-2015 agenda. En S. McGrath, \& Q. Gu (Eds.), Routledge Handbook of International Education and Development (pp. 62-77). New York: Routledge. 
Denzin, N. K., \& Lincoln, Y. S. (2012). Manual de investigación cualitativa. Barcelona: Gedisa.

Domínguez, N. (2015). Estrategia para la gestión de la profesionalización docente en la carrera de Medicina (Tesis de máster en Educación Superior). Universidad de Ciego de Ávila, Ciego de Ávila.

Donoso-Vázquez, T., Montané, A., \& Pessoa, M. E. (2014). Género y calidad en Educación Superior. Revista Electrónica Interuniversitaria de Formación del Profesorado, 17(3), 157-171.

Donoso-Vázquez, T., \& Velasco-Martínez, A. (2013). ¿Por qué una propuesta de formación en perspectiva de género en el ámbito universitario? Profesorado. Revista de Currículum y Formación del Profesorado, 17(1), 71-88.

Foster, E., Kerr, P., Hopkins, A., Byrne, C., \& Ahall, L. (2013). The personal is not political: At least in the UK's top politics and IR departments. British Journal of Politics and International Relations, 15(4), 566-585.

García-Pérez R., Rebollo Ma A., Vega, L., Barragán-Sánchez, R., Buzón, O., \& Piedra J. (2011). El patriarcado no es transparente: Competencias del profesorado para reconocer desigualdad. Cultura y Educación, 23(3), 385-397.

Giraldo, E., \& Colyar, J. (2012). Dealing with gender in the classroom: A portrayed case study of four teachers. International Journal of Inclusive Education, 16(1), 25-38.

Glaser, B., \& Strauss, A. (1967). The discovery of Grounded Theory. New York: Aldine Publishing Company.

Grünberg, L. (2011). From gender studies to gender IN studies and beyond. In L. Grünberg (Ed.), From gender studies to gender IN studies: Case studies on gender-inclusive curriculum in Higher Education (pp. 7-15). Bucharest: UNESCO-CEPES.

Huber, L. (2012). AQUAD Seven. Manual for the analisis of qualitative data. Tübingen, Ingeborg Huber Verlag.

Kabeer, N. (2005). Gender equalityand women'sempowerment: A critical analysis of the third millennium development goal 1. Gender \& Development, 13(1), 13- 24.

Ley Orgánica $3 / 2007$, de 22 de marzo, para la igualdad efectiva de mujeres y hombres. Boletín Oficial del Estado, número 131 de 02/06/2011. Recuperado de http://www.boe.es/aeboe/consultas/bases_datos/ doc. php?id=BOE-A-2007-6115

Maykut, P., \& Morehouse, R. (1994). Investigación cualitativa. Una guía práctica y filosófica. Barcelona: Hurtado.

Martínez, M. A., Merma, G., \& Gavilán, D. (2013). Redes colaborativas de profesorado: proyecto Diseño y atención a las oportunidades de género en la Educación Superior. RIIEP, 6(2), 161-170.

McNeil, Maureen (1992). Pedagogical praxis and problems: Reflections on teaching about gender relations. In $\mathrm{H}$. Hillary, A. Phoenix, \& J. Stacey (Eds.), Working out: New directions for women's studies. London/ Washington: The Falmer Press.

Merma-Molina, G., Ávalos, M. A., \& Martínez, M. A. (2015). La relevancia encubierta del género: las percepciones de los futuros maestros sobre la igualdad y las desigualdades contextuales. La Manzana de la Discordia, 10(2), 93-104.

Merma-Molina, G., Ávalos, M. A., \& Martínez, M. A. (2017). La igualdad de género en la docencia universitaria. Percepciones del alumnado. La Manzana de la Discordia, 12(1), 103-115.

Miranda, O. (2016). La profesionalización docente del médico profesor (Tesis doctoral). Universidad de Oriente, Santiago de Cuba.

Naranjo, A. (2010). La transversalización del enfoque de género en la formación de profesionales de la educación, desde las potencialidades de la disciplina Formación Pedagógica General (Tesis doctoral). Universidad de Ciencias Pedagógicas José de la Luz y Caballero, Holguín.

Patton, M. (1990). Purposeful sampling. Qualitative evaluation and research methods, 2, 169-186.

Pulido, E. (2014). Sistema de procedimientos metodológicos de evaluación del impacto del proceso de profesionalización docente en la Universidad de Ciego de Ávila (Tesis de máster). Universidad de Ciego de Ávila, Ciego de Ávila.

Rebollo, M. Á., García, R., Piedra, J., \& Vega, L. (2011). Diagnóstico de la cultura de género en educación: actitudes del profesorado hacia la igualdad. Revista de Educación, (355), 521-526.

Santos, B. de S. (2012). Public sphere and epistemologies of the South. Africa Development, 37, 1, 43-67.

Santos, B., Nunes, J. A., \& Meneses, M. P. (2007). Opening up the canon of knowledge and recognition of difference. In B. S. Santos (Ed.), Another Knowledge is Possible: Beyond Northern Epistemologies (pp. VII-XVIX). London: Verso.

Tembon, M., \& Fort, L. (2008). Girls' Education in the 21st century: Gender equality, empowerment, and economic growth. Washington DC: World Bank.

Unterhalter, E. (2014). Measuring education for the Millennium Development Goals: Reflections on targets, indicators, and a post-2015 framework. Journal of Human Development and Capabilities, 15(1) 1-12. 